\title{
LETTER
}

\section{Cavity-assisted ultrafast long-range periodic energy transfer between plasmonic nanoantennas}

\author{
Martin Aeschlimann ${ }^{1}$, Tobias Brixner ${ }^{2,3}$, Mirko Cinchetti ${ }^{4}$, Benjamin Frisch ${ }^{1}$, Bert Hecht ${ }^{3,5}$, Matthias Hensen ${ }^{2,6}$, \\ Bernhard Huber ${ }^{2}$, Christian Kramer ${ }^{2}$, Enno Krauss ${ }^{5}$, Thomas H Loeber ${ }^{7}$, Walter Pfeiffer ${ }^{6}$, Martin Piecuch ${ }^{1}$ and \\ Philip Thielen ${ }^{1,8}$
}

Light: Science \& Applications (2017) 6, e17111; doi:10.1038/lsa.2017.111; published online 3 November 2017

Radiationless energy transfer is at the core of diverse phenomena, such as light harvesting in photosynthesis ${ }^{1}$, energy-transfer-based microspectroscopies ${ }^{2}$, nanoscale quantum entanglement ${ }^{3}$ and photonic-mode hybridization ${ }^{4}$. Typically, the transfer is efficient only for separations that are much shorter than the diffraction limit. This hampers its application in optical communication and quantum information processing, which require spatially selective addressing. Here, we demonstrate highly efficient radiationless coherent energy transfer over a distance of twice the excitation wavelength by combining localized and delocalized ${ }^{5}$ plasmonic modes. Analogous to the Tavis-Cummings model, two whispering-gallery-mode antennas ${ }^{6}$ placed in the foci of an elliptical plasmonic cavity ${ }^{7}$ fabricated from single-crystal gold plates act as a pair of oscillators coupled to a common cavity mode. Time-resolved two-photon photoemission electron microscopy (TR 2P-PEEM) reveals an ultrafast long-range periodic energy transfer in accordance with the simulations. Our observations open perspectives for the optimization and tailoring of mesoscopic energy transfer and long-range quantum emitter coupling.

Surface plasmon polaritons (SPPs), that is, coherent modes of collective electronic motion and electromagnetic fields, constitute building blocks for ultrafast on-chip routing of signals and energy, 8,9 because they allow the concentration of fields to typical dimensions of microelectronics ${ }^{10,11}$. In particular, one-dimensional plasmonic waveguides $^{12}$ could be used to enhance energy transfer between spatially separated quantum emitters ${ }^{13}$, to coherently control energy flow partitioning into different output ports ${ }^{14}$ in all-optical nanocircuitry, and even to transmit quantum information ${ }^{15}$ in future nanoscale quantum technologies. For nanoscale quantum information processing, it is essential but challenging to establish long-range strong coupling between spatially well-separated quantum emitters.
How can this be achieved? Both large local field enhancements at the positions of the quantum emitters and long-range energy transport with little loss are desirable. Localized surface plasmon (LSP) modes yielding sufficiently large field enhancements and strong coupling of single quantum emitters to such a field mode have recently been demonstrated ${ }^{16}$. However, efficient long-range energy transfer is hindered by this field localization and the typically rather strong LSP damping. In contrast, waveguide modes or delocalized SPPs alone do not reach sufficiently large field enhancement. As demonstrated here, the hybridization of localized and delocalized modes allows the optimization of local coupling and facilitates periodic, SPP-mediated energy transfer between two nanoantennas over a distance of twice the excitation wavelength.

In our device, two whispering-gallery-mode (WGM) resonators ${ }^{6,17}$ act as plasmonic nanoantennas (Figure 1a). They are placed in the foci of an elliptical SPP cavity ${ }^{7}$ to achieve enhanced coupling, similar to the scheme demonstrated for Fabry-Pérot microcavities ${ }^{18}$. Strong coupling of the plasmonic nanoantennas is realized by spectrally overlapping the $m=0$ mode of the WGM antennas ${ }^{6}$ (Supplementary Fig. S1b) with the standing-wave cavity resonance (Supplementary Fig. S1a). Structural parameters are given in the caption of Figure 1. As expected in the strong coupling regime, FDTD simulations reveal a periodic oscillation of field energy between both WGM antennas upon selective excitation of one antenna $\left(\mathrm{WGM}_{r}\right)$ with a 30 -fs pulsed dipole source placed inside the mesa-shaped part (Figure 1b). The cavitymediated oscillatory excitation transfer from $\mathrm{WGM}_{r}$ to the left antenna $\mathrm{WGM}_{l}$ and back occurs with a period of $86 \mathrm{fs}$. Two full oscillations are observed, demonstrating that indeed strong coupling between the WGM antennas is achieved. Taking the local near-field intensity as a measure of the energy content of the antenna modes and

\footnotetext{
${ }^{1}$ Fachbereich Physik and Research Center OPTIMAS, University of Kaiserslautern, Erwin-Schrödinger-Str. 46, Kaiserslautern 67663, Germany; ${ }^{2}$ Institut für Physikalische und Theoretische Chemie, Universität Würzburg, Am Hubland, Würzburg 97074, Germany; ${ }^{3}$ Röntgen Research Center for Complex Material Systems (RCCM), Universität Würzburg, Am Hubland, Würzburg 97074, Germany; ${ }^{4}$ Experimentelle Physik VI, Fakultät Physik, Technische Universität Dortmund, Dortmund 44221, Germany; ${ }^{5}$ Nano-Optics and BioPhotonics Group, Department of Experimental Physics 5, Universität Würzburg, Am Hubland, Würzburg 97074, Germany; ${ }^{6}$ Fakultät für Physik, Universität Bielefeld, Universitätsstr. 25, Bielefeld 33615, Germany; ${ }^{7}$ Nano Structuring Centre and Research Center OPTIMAS, Erwin-Schrödinger-Str. 13, Kaiserslautern 67663, Germany and ${ }^{8}$ Graduate School of Excellence Materials Science in Mainz, Gottlieb-Daimler-Str. 47, Kaiserslautern 67663, Germany

Correspondence: W Pfeiffer, Email: pfeiffer@physik.uni-bielefeld.de

Received 10 February 2017; revised 14 July 2017; accepted 16 July 2017
} 
omitting the excitation time window yields an energy transfer efficiency between the antennas of $\sim 7 \%$.

The strong coupling between the WGM antennas could serve to more efficiently transfer energy between two quantum emitters placed above both antennas. The normalized energy transfer rate (nETR)

a

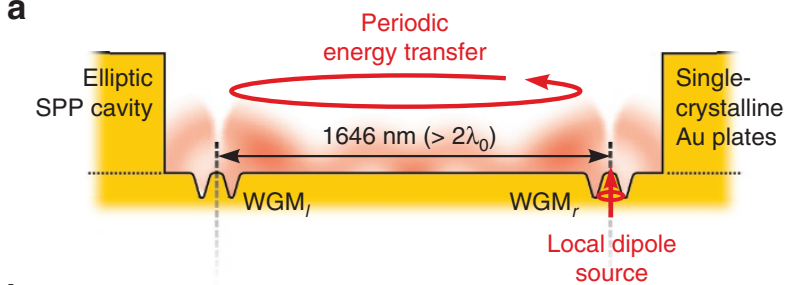

b

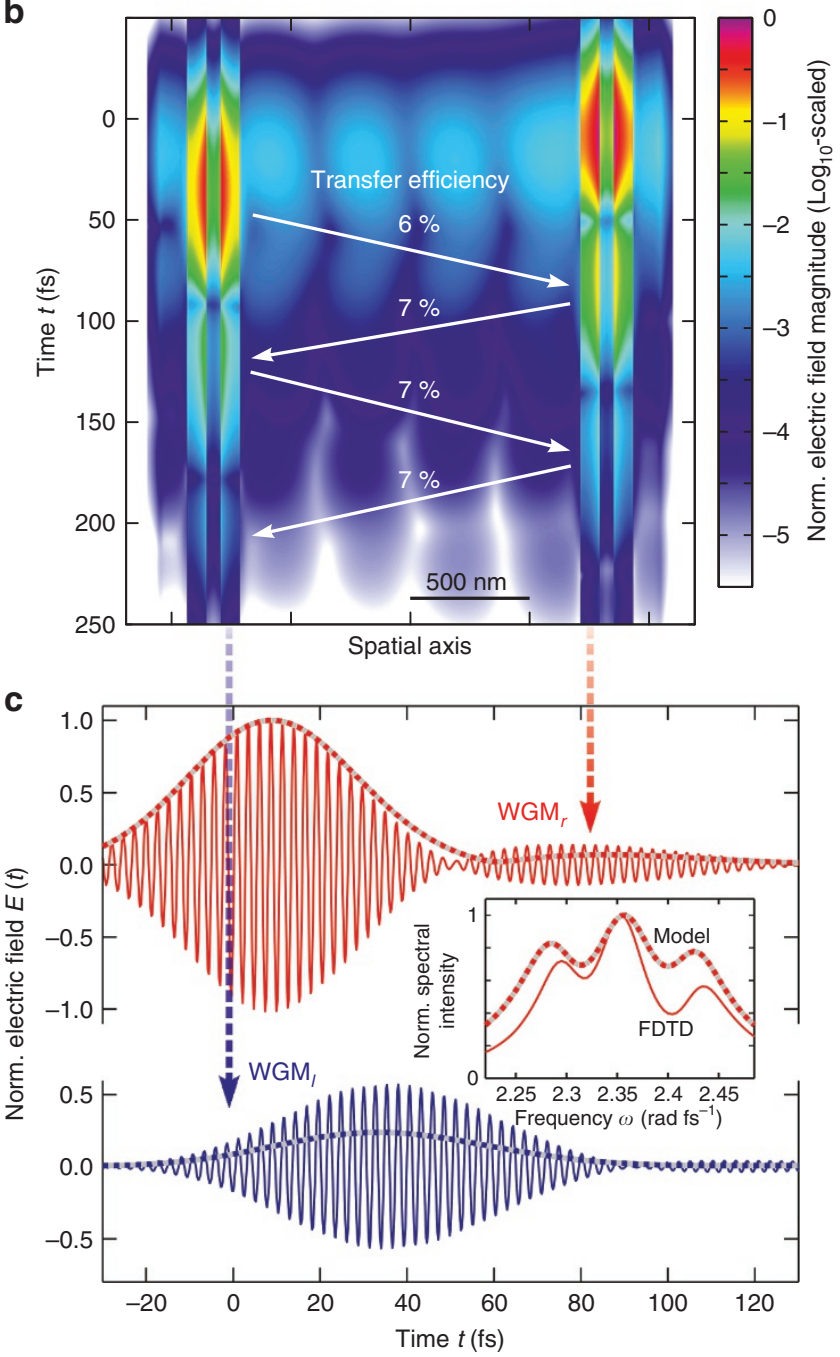

d

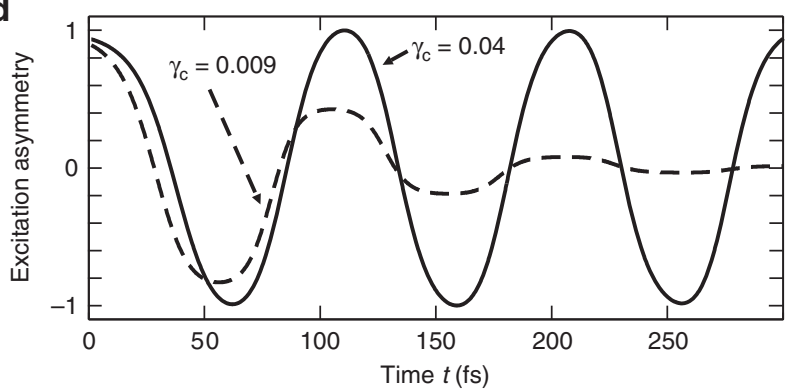

serves to compare this efficiency for different optical near-field assisted schemes. It is defined as the energy transfer rate between two emitters placed in a particular near-field mode divided by the rate obtained in vacuum for the same emitter separation. From simulations based on the FDTD method and the formalism described in Ref 19, we obtain a $\mathrm{nETR} \approx 10^{7}$ for resonant emitters $\left(2.356 \mathrm{rad} \mathrm{fs}^{-1}, 800 \mathrm{~nm}\right)$ located $10 \mathrm{~nm}$ above the antennas. In a Förster resonant energy transfer (FRET), two dipole emitters reach the same transfer rate for $15 \mathrm{~nm}$ separation ${ }^{20}$. Hence, the two emitters act effectively as a donoracceptor pair despite their large separation. Note that the nETR at $800 \mathrm{~nm}$ increases slightly as the cavity $Q$-factor is reduced from $Q=173$ to $Q=42$ by lowering the cavity wall height, whereas the nETR drops slightly for other wavelengths. In general, the impact of $Q$ on the nETR is small, and the benchmark value of $n E T R \approx 10^{7}$ holds. To the best of our knowledge, the highest nETR reported to date over a similar distance relies on one-dimensional plasmonic waveguides and is approximately two orders of magnitude smaller ${ }^{13}$ than that of our design. Hence, as discussed below, WGM antennas embedded in an SPP cavity represent an excellent possibility to realize SPP-mediated strong coupling between single quantum emitters $^{21}$.

The device's working principle leading to periodic energy transfer is well captured by a coupled-oscillator model (Supplementary Information Section 2). This classical analogue of the Tavis-Cummings model ${ }^{22}$, that is, the interaction of emitters via a common cavity mode, accurately describes the local fields at both antennas (Figure 1c, see Supplementary Information Section 2 for the frequency-domain representation). Interestingly, the model implies that for given antennas, the cavity damping constant $\gamma_{c}$ controls the ultrafast energy redistribution between them in a rather counterintuitive manner (Figure 1d). For a low cavity damping $\gamma_{\mathrm{c}}$, the excitation asymmetry, that is, the energy difference between $\mathrm{WGM}_{l}$ and $\mathrm{WGM}_{r}$ normalized to the total energy of both modes, exhibits a fast energy equipartition between both antennas (Figure 1d, dashed line), whereas for a higher $\gamma_{\mathrm{c}}$, the excitation asymmetry continues to oscillate (Figure 1d, solid line). In the latter case, the amounts of cavity and antenna loss converge, which leads to an optimized energy

Figure 1 Concept and simulation of periodic coherent energy transfer between localized modes embedded in a plasmonic cavity. (a) Cross section along the major axis of an elliptical surface plasmon polariton (SPP) cavity (2.092 $\mu \mathrm{m}$ major axis length, $1.450 \mu \mathrm{m}$ minor axis length, $500 \mathrm{~nm}$ rim height). Two whispering-gallery-mode (WGM) antennas are embedded at the two focal points of the cavity. The right antenna (WGM $\left.{ }_{r}\right)$ is locally excited by a dipole source (red arrow) emitting a $30 \mathrm{fs}$ Gaussian pulse (center frequency $\omega_{\mathrm{L}}=2.41 \mathrm{rad} \mathrm{fs}^{-1}$ ). The diameter and groove depth $d_{\mathrm{gr}}$ of the WGM antennas are $140 \mathrm{~nm}$ and $118 \mathrm{~nm}$, respectively. (b) Temporal evolution of the electric field magnitude recorded along the major axis of the ellipse and in a plane $10 \mathrm{~nm}$ below the cavity floor as a function of the time $t$ after the excitation pulse. The percentages close to the white arrows indicate the energy transfer efficiencies, as deduced from the square modulus of the local field amplitude. (c) Local fields at the two WGMs as a function of time $t$. The origins of the dashed arrows indicate the positions at which the local fields are recorded, that is, in the grooves of the WGM antennas. The dashed lines show the corresponding field magnitudes derived from the coupled-oscillator model with adjusted parameters. The spectral responses for $\mathrm{WGM}_{r}$ (excited antenna) derived from the FDTD (solid line) and coupled-oscillator model (dashed line) are shown in the inset. (d) Impact of cavity damping $\gamma_{c}$ on the temporal evolution of the excitation asymmetry between $\mathrm{WGM}_{r}$ and the left antenna

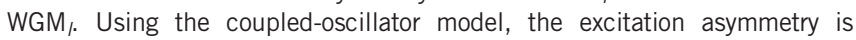
calculated as the field intensity difference between both antennas normalized to the sum of both field intensities. 
transfer. The underlying effect is called impedance matching and is well known in the field of plasmonics ${ }^{23}$ and physics in general ${ }^{24}$. This qualitative behavior is also confirmed by FDTD simulations for different cavity wall heights (Supplementary Information Section 3) and demonstrates the utility of the structure as a model system for investigating energy transfer processes.

Limitations of this cavity-based energy transfer scheme were assessed by FDTD and coupled-oscillator simulations. The maximum distance between the antennas for maintaining the demonstrated coupling scheme is limited to approximately $4 \mu \mathrm{m}$ because at that point, neighboring cavity modes start overlapping with the WGM resonance, leading to a more complicated hybridization scheme, which is not explored here. Note that this maximum distance increases if narrower antenna resonances are employed. The strong coupling regime will break down when the hybridized modes (Figure 1c, inset) can no longer be resolved. This would occur if the cavity damping increases due to, for example, increased cavity wall leakage and $Q<30$ is reached.

For the experimental device implementation, we use chemically synthesized single-crystalline Au plates ${ }^{25,26}$ to avoid loss channels such as grain boundaries (Figure 2a). The cavity is prepared by stacking a plate with an elliptical focused ion beam cutout on top of a pristine $\mathrm{Au}$ plate, resulting in an atomically smooth cavity floor and steep side walls. Then, focused ion beam milling is used to cut the circular grooves of the WGM antennas. A scanning electron microscopy image of a complete cavity system is shown in Figure $2 \mathrm{~b}$.

For TR 2P-PEEM ${ }^{27}$, the structure is illuminated by two ultrashort laser pulses with a variable time delay $\Delta t$ (Figure $2 \mathrm{~b}$ ) introduced by a phase-stabilized Mach-Zehnder interferometer. $\mathrm{WGM}_{r}$ is preferentially excited because the cavity rim (height $440 \mathrm{~nm}$ ) shades $\mathrm{WGM}_{l}$ for grazing incidence. Photoelectron emission patterns of three cavities with varying geometries are recorded upon single-pulse excitation using PEEM and are shown in Figure 3a. For WGM antennas with a groove depth of $d_{\mathrm{gr}}=90 \mathrm{~nm}$, the emission pattern is dominated by the emission from these resonant antennas (Figure 3a, left and right panels), and two strong emission spots reveal information about the field at both antennas. Varying $d_{\mathrm{gr}}$ shifts the resonance, and for $d_{\mathrm{gr}}=110 \mathrm{~nm}$, the excitation is no longer resonant (Figure $3 \mathrm{a}$, middle panel). Furthermore, the emission pattern reveals that the stillresonant excitation of the elliptical cavity has a negligible effect on the photoemission yield since there is very weak emission and no sign of a photoelectron emission cavity mode pattern. Note that monitoring the energy flow through the cavity by PEEM is not possible because of this low yield from the cavity mode. To fine-tune the resonance condition for the cavity mode, we varied the cavity's major axis length by $20 \mathrm{~nm}$ from the left to the right panel. According to FDTD simulations, this changes the cavity resonance position by only $0.7 \%$. The slight reduction of the peak emission yield compared with the emission pattern shown in the left panel of Figure $3 \mathrm{a}$ indicates that the resonance conditions for the cavity are not perfectly met. The total yield for single-pulse excitation is used as an indicator for resonant excitation; thus, we employ the structure shown in the left panel of Figure $3 \mathrm{a}$ in all further investigations.

TR 2P-PEEM patterns of the two WGM antennas were recorded as a function of the delay $\Delta t$ between the two fs laser pulses ( $24 \mathrm{fs}$ pulse duration). The local emission yield as a function of $\Delta t$ (Figure $3 \mathrm{~b}$ ), that is, the nonlinear two-pulse correlation signal, contains information about the local spatiotemporal field evolution ${ }^{27}$ : For $\Delta t>20 \mathrm{fs}$, the pulses are fairly well separated, and the signals of the excited and shaded WGM antennas differ significantly. The envelope of the correlation signal of the excited antenna develops a constriction at a

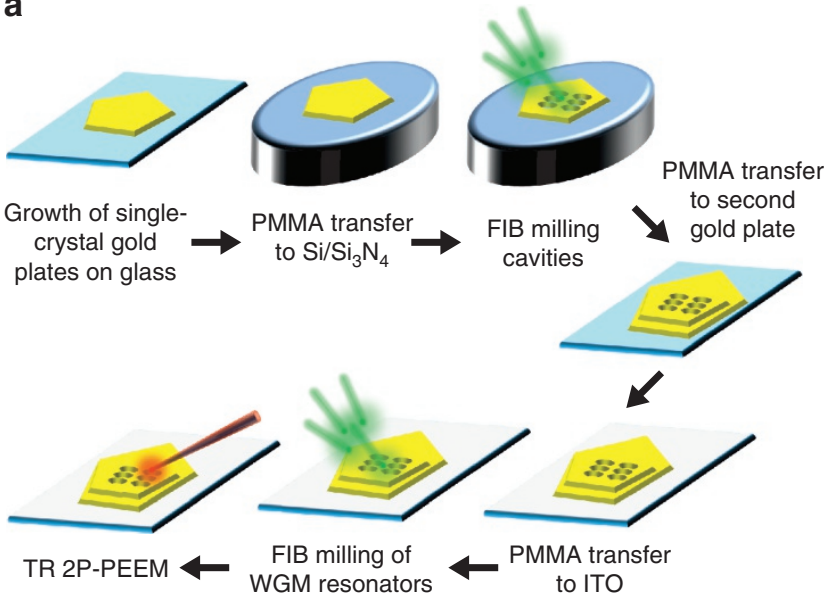

b

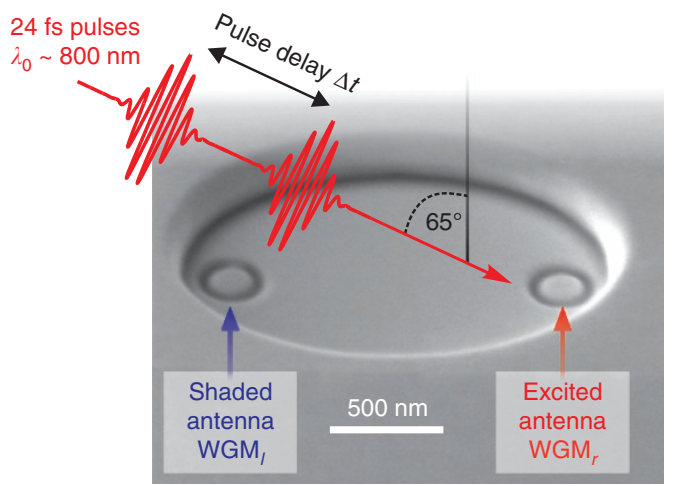

Figure 2 Preparation of high-Q SPP cavities with embedded WGM antennas. (a) Preparation steps for cavity structures based on single-crystalline $\mathrm{Au}$ plates (for details, see Methods) and poly(methyl methacrylate)-assisted (PMMA) transfer steps. All nanostructuring is done by focused ion beam (FIB) milling. Stacking of two Au plates avoids ion bombardment damage of the cavity floor and is essential to achieve high-Q SPP cavities. (b) Scanning electron microscopy (SEM) image of a representative cavity with embedded WGM antennas (SEM column is tilted by $52^{\circ}$ with respect to the surface normal). In addition, the illumination condition is indicated for TR 2P-PEEM as used to experimentally observe the periodic energy transfer between the WGM antennas. A sequence of two ultrashort laser pulses with variable delay $\Delta t$ is directed onto the sample with an incident angle of $65^{\circ}$. The electrons emitted from the cesiated Au surface in a two-photon process are recorded using PEEM (not shown).

$\Delta t \approx 40 \mathrm{fs}$, whereas the envelope of the shaded antenna exhibits a continuous decrease. Note that both signals are recorded simultaneously and therefore directly reflect differences in the temporal evolution of the local fields. The measured correlation signals are again well reproduced by the coupled-oscillator model (black lines, Figure $3 \mathrm{~b}$ ) if it is considered that the shaded antenna receives some direct excitation (see the caption of Figure 3 for fit parameters). Even the subtle phase effects in the oscillatory correlation signal match (Supplementary Information Section 4). The slightly lifted wings in the experimental two-pulse correlation curves, which are not reproduced by our model, arise from a small amount of uncompensated group velocity dispersion of the laser pulses.

On the basis of the adjusted model parameters, one can calculate the time-domain responses of both antennas upon single-pulse excitation (Figure 3c). The directly excited antenna shows a strong response during the rising slope of the excitation pulse centered at $t=0 \mathrm{fs}$, whereas at the same time, the shaded antenna oscillates with 

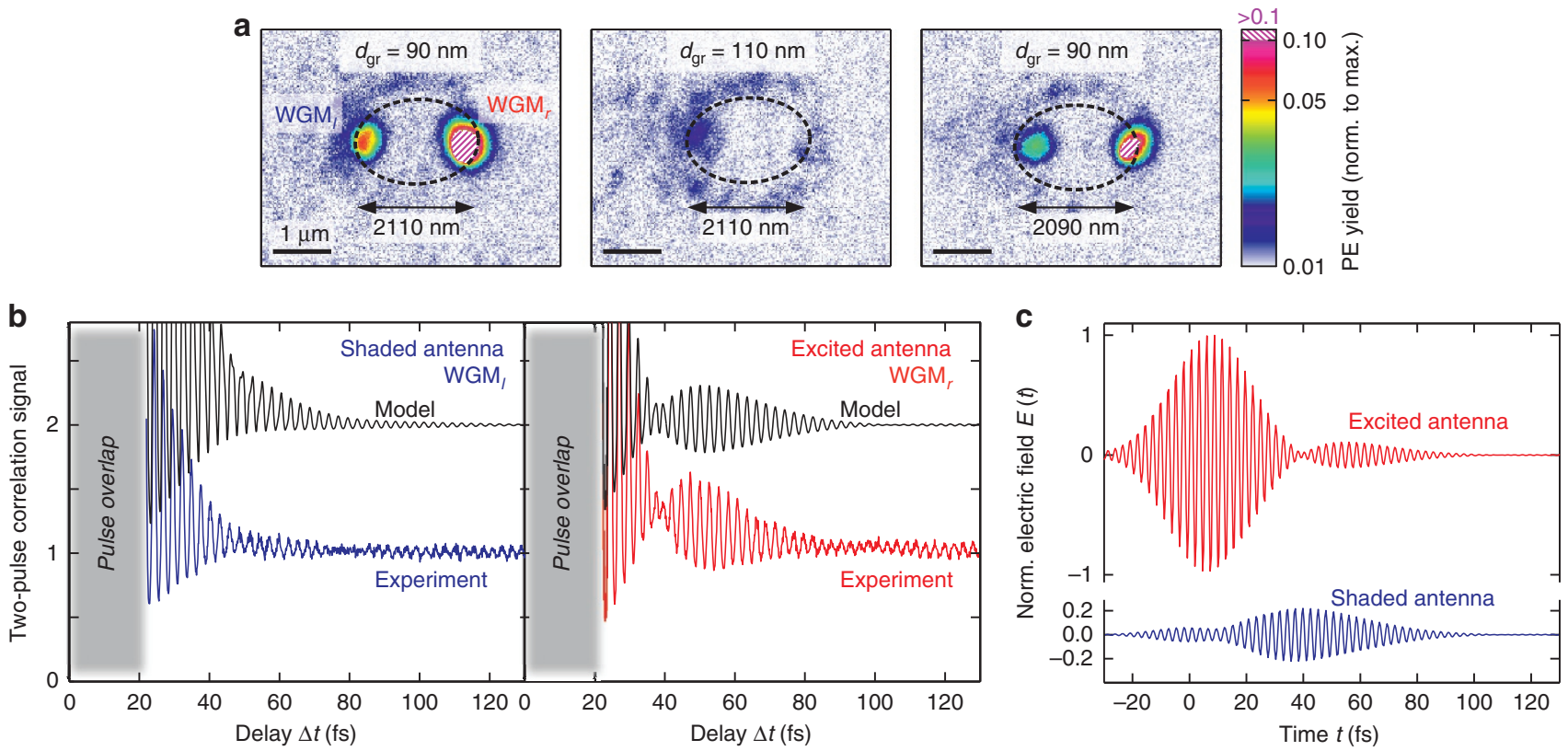

Figure 3 Experimental demonstration of periodic long-range energy transfer. (a) Two-photon photoemission pattern of three elliptical SPP cavities with varying geometric parameters. All embedded WGMs have a diameter of $250 \mathrm{~nm}$ and the indicated groove depth $d_{\mathrm{gr}}$. The dashed ellipses indicate the rims of the plasmonic cavities. (b) Two-pulse correlation photoemission yields for excited (right panel, red line) and shaded (left panel, blue line) antennas as a function of delay $\Delta t$ between the two exciting laser pulses. The signals are integrated over $20 \times 20$ pixel arrays centered on the WGM-related emission hot spots. The yields are normalized to the yield obtained for large $\Delta t$. Normalized two-pulse correlation signals derived from a coupled-oscillator model with adjusted model parameters are shown for both antennas as black lines (moved upwards by one unit for better visibility). Model parameters: degenerate resonance frequencies $\omega_{l, r, c}=2.33 \mathrm{rad} \mathrm{fs}^{-1}$ for left and right WGMs and the cavity, damping parameters $\gamma_{1, \mathrm{r}}=0.078 \mathrm{fs}^{-1}$ for left and right WGMs and $\gamma_{\mathrm{c}}=0.022 \mathrm{fs}{ }^{-1}$ for the cavity, coupling constant $\kappa=0.16 \mathrm{rad}^{2} \mathrm{fs}^{-2}$ or WGM-cavity coupling, fraction of incident field strength directly delivered to the shaded WGM antenna $\alpha=0.11$ and relative excitation phase $\varphi=-\pi / 10$ for both antennas. (c) Temporal evolution of the electric fields of both WGM antennas upon single-pulse excitation based on the coupled-oscillator model adjusted to the experimental correlation signals shown in (b).

an amplitude that is 10 times lower, that is, a direct excitation intensity that is 100 times weaker. At $t \approx 40 \mathrm{fs}$, the oscillation of the excited antenna vanishes, and the shaded antenna exhibits its maximum oscillation amplitude. At $t \approx 60 \mathrm{fs}$, the excited antenna shows the second maximum of the oscillation amplitude, thus indicating the completion of the first full period of the oscillatory energy exchange. The ratio of 0.1 between the first and second maxima of the excitedantenna oscillation amplitude corresponds to an efficiency of $0.1^{2}=1 \%$ for a full period of energy exchange. Hence, the experimentally realized energy transfer efficiency between the antennas is $\sim 10 \%$ and agrees well with the theoretical $7 \%$ efficiency obtained in FDTD simulations. Note that the predicted theoretical efficiency does not necessarily reflect optimum coupling conditions since we did not explore the full parameter space, including the exact antenna position, its diameter, the groove depth or its width. Furthermore, the experimental efficiency is retrieved from the phenomenological coupled-oscillator model, and small deviations from a full electrodynamic simulation are expected. We would also like to stress that the more efficient initial transfer seen in the FDTD simulation (Figure 1b), which is based on the direct excitation of the cavity caused by the dipole source, is irrelevant here: as estimated from far-field simulations, this cavity mode excitation is $\sim 10-20$ times less efficient compared to the WGM antenna excitation.

In summary, we have designed and demonstrated a plasmonic device that enables long-range strong coupling between selectively addressable nanoantennas separated by approximately twice the excitation wavelength, thus significantly exceeding the distance of approximately ten nanometers that is required for similarly efficient free-space coupling. The dynamics are well described by a coupled- oscillator model, which shows complex energy flow dynamics that can be tailored via dissipation of involved plasmonic resonances. In addition to possible applications of our scheme in ultrafast nanocircuitry, the presented system is a highly interesting scheme for coupling spatially separated quantum emitters: energy transfer rates are demonstrated that are two orders of magnitude larger compared to the $1 \mathrm{D}$ plasmonic waveguides ${ }^{13}$ that were proposed for strong quantum emitter coupling ${ }^{28}$ over large distances ${ }^{3}$. Recently, strong coupling between a single quantum emitter and a plasmonic resonator has been demonstrated ${ }^{16}$. On the basis of this finding, our plasmonic hybridization scheme provides a direct approach for achieving a strong coupling of quantum emitters over large separations because the coupling is transitive for properly chosen emitter parameters; that is, if $\mathrm{A}$ and $\mathrm{B}$, and $\mathrm{B}$ and $\mathrm{C}$ are strongly coupled, $\mathrm{A}$ and $\mathrm{C}$ are also strongly coupled. Note that the demonstrated hybridization scheme reduces the linewidth of the antenna mode and thus facilitates more pronounced strong-coupling-induced mode splitting.

\section{METHODS \\ FDTD calculations}

For FDTD calculations, the commercial software Lumerical (Lumerical Solutions, Inc., Vancouver, Canada, Version 8.11.337) is used based on the data of Johnson and Christy ${ }^{29}$. The elliptical cavity is meshed adaptively such that the effective wavelength inside a specific material is resolved by 22 grid points. Locally, extra polygon meshes with a 2-nm resolution are employed to model the WGM antennas with rounded V-shaped grooves. Dipole sources placed within the structure (on the symmetry axis of the WGM antenna $38 \mathrm{~nm}$ below the Au-vacuum interface) are used for excitation. Tests show that the 
periodic energy transfer between the WGM antennas is unaffected by whether the source is placed inside or outside the metal. Perfectly matched layer boundary conditions are chosen, and the symmetry plane defined by the major axis of the elliptical cavity and the surface normal is exploited to reduce the memory demand. Note that simulations with and without WGM antennas are performed using identical meshes. For cavity simulations without WGM antennas, the dipole source is located $10 \mathrm{~nm}$ above the cavity floor. Frequencydomain monitors are used to record the response function in the vicinity of the WGM antennas and the cavity mode.

The normalized energy transfer rate (nETR) is derived from FDTD simulations in which the dipole source is placed $10 \mathrm{~nm}$ above one WGM antenna. Following the strategy that was introduced by Marocico et $a l^{19}$, and employed by Martin-Cano et al ${ }^{13}$, the nETR is obtained as the ratio between the intensity (absolute magnitude squared of the induced local electric field) $10 \mathrm{~nm}$ above the other antenna and the intensity at the same position but for a dipole emitting in vacuum. In Lumerical FDTD, the electric field strength is referenced to the analytical formula describing the radiated power of an electric dipole inside a homogeneous material ${ }^{20}$. Since the dipole source itself is located in vacuum in both simulations, that is, with and without plasmonic enhancement, the obtained field data are referenced to the same analytical source spectrum.

\section{Preparation of elliptical SPP cavity with embedded WGM nanoantennas}

The demonstrated efficient energy transfer between plasmonic antennas relies on low damping of the involved resonances. To meet this requirement, a novel preparation scheme is employed (Figure 2a). First, single-crystalline gold plates are chemically synthesized on a glass substrate $\mathrm{e}^{26}$ and then transferred onto a conductive substrate by a poly (methyl methacrylate) (PMMA)-assisted transfer method ${ }^{30}$. Using FIB, one gold plate is perforated with elliptical holes of desired major and minor axis lengths $a$ and $b$. Using the PMMA transfer again, the perforated gold plate is then stacked on top of another untreated chemically synthesized gold plate. The lower gold plate serves as an atomically flat cavity floor, and the elliptical holes of the upper gold plate serve as cavity walls. The wall height is controlled by selecting plates with the proper thickness after the growth process. As a last step, the elliptical plasmonic cavities are transferred to an indium tin oxide (ITO) substrate and WGM nanoantennas are milled into the floor via FIB milling. The ITO substrate avoids effects of sample charging during PEEM experiments. The device geometries for the cavity and WGM antennas were optimized to match their spectral resonances to the excitation wavelength.

\section{Time-resolved two-photon photoelectron emission microscopy (TR 2P-PEEM)}

We use a Ti:Sapphire laser oscillator (Newport Spectra-Physics Tsunami, $24 \mathrm{fs}, 80 \mathrm{MHz}, 796 \mathrm{~nm}, 9 \mathrm{~nJ}$ maximum energy per pulse) to generate a sequence of two ultrashort laser pulses with a variable delay $\Delta t$ by using a home-built phase-stabilized Mach-Zehnder interferometer. The pulses are focused onto the sample $(20 \mathrm{~cm}$ focal length, $50 \mu \mathrm{m}$ focus diameter) under $65^{\circ}$ angle of incidence. Prior to measurements, the sample is cesiated to lower the work function of the surface, thus enabling a two-photon photoemission process from Au. For each time delay $\Delta t$, the spatial distribution of the electron emission is recorded by the CCD camera of the PEEM (Focus GmbH, Huenstetten, Germany) with a spatial resolution of better than $40 \mathrm{~nm}$.

\section{CONFLICT OF INTEREST}

The authors declare no conflict of interest.

\section{AUTHOR CONTRIBUTIONS}

MA, TB, BHe and WP initiated and supervised the work. $\mathrm{MH}$ performed the FDTD calculations and developed the coupled-oscillator model. BF, MH, PT, EK and ThHL designed the nanostructure and prepared the sample. MC, BF, $\mathrm{MH}, \mathrm{BHu}, \mathrm{CK}, \mathrm{MP}$ and PT performed the time-resolved PEEM experiments. $\mathrm{BF}, \mathrm{MH}$ and $\mathrm{CK}$ performed the data analysis. All authors contributed to manuscript preparation.

\section{ACKNOWLEDGEMENTS}

This work was supported by the German Science Foundation (DFG) within the SPP 1391 (MA, TB, WP, BHe and EK), the GSC 266 (PT), and the ERC Consolidator Grant MULTISCOPE (TB). We wish to thank Sandra Wolff and Bert Lägel of the Nano Structuring Center for their expertise and support during the sample preparation.

1 Collini E, Wong CY, Wilk KE, Gurmi PMG, Brumer $\mathrm{P}$ et al. Coherently wired light-harvesting in photosynthetic marine algae at ambient temperature. Nature 2010; 463: 644-647.

2 Jares-Erijman EA, Jovin TM. FRET imaging. Nat Biotechnol 2003; 21: 1387-1395.

3 González-Tudela A, Martín-Cano D, Moreno E, Martín-Moreno L, Tejedor C et al. Entanglement of two qubits mediated by one-dimensional plasmonic waveguides. Phys Rev Lett 2011; 106: 020501.

4 Aeschlimann M, Brixner T, Fischer A, Kramer C, Melchior P et al. Coherent twodimensional nanoscopy. Science 2011; 333: 1723-1726.

5 de Torres J, Ferrand P, Colas des Francs G, Wenger J. Coupling emitters and silver nanowires to achieve long-range plasmon-mediated fluorescence energy transfer. ACS Nano 2016; 10: 3968-3976.

6 Vesseur EJR, Polman A. Plasmonic whispering gallery cavities as optical nanoantennas. Nano Lett 2011; 11: 5524-5530.

7 Schoen DT, Coenen T, García de Abajo FJ, Brongersma ML, Polman A. The planar parabolic optical antenna. Nano Lett 2013; 13: 188-193.

8 Brongersma ML, Shalaev VM. The case for plasmonics. Science 2010; 328 : 440-441.

9 Zia R, Schuller JA, Chandran A, Brongersma ML. Plasmonics: the next chip-scale technology. Mater Today 2006; 9: 20-27.

10 Schuller JA, Barnard ES, Cai WS, Jun YC, White JS et al. Plasmonics for extreme light concentration and manipulation. Nat Mater 2010; 9: 193-204.

11 Gramotnev DK, Bozhevolnyi SI. Plasmonics beyond the diffraction limit. Nat Photonics 2010; 4: 83-91.

12 Bozhevolnyi SI, Volkov VS, Devaux E, Laluet J-Y, Ebbesen TW. Channel plasmon subwavelength waveguide components including interferometers and ring resonators. Nature 2006; 440: 508-511.

13 Martín-Cano D, Martín-Moreno L, García-Vidal FJ, Moreno E. Resonance energy transfer and superradiance mediated by plasmonic nanowaveguides. Nano Lett 2010; 10: 3129-3134.

14 Rewitz C, Razinskas G, Geisler P, Krauss E, Goetz S et al. Coherent control of plasmon propagation in a nanocircuit. Phys Rev App/ 2014; 1: 014007.

15 Kumar S, Kristiansen NI, Huck A, Andersen UL. Generation and controlled routing of single plasmons on a chip. Nano Lett 2014; 14: 663-669.

16 Chikkaraddy R, de Nijs B, Benz F, Barrow SJ, Scherman OA et al. Single-molecule strong coupling at room temperature in plasmonic nanocavities. Nature 2016; 535: 127-130.

17 Vesseur EJR, García de Abajo FJ, Polman A. Modal decomposition of surface-plasmon whispering gallery resonators. Nano Lett 2009; 9: 3147-3150.

18 Ameling R, Giessen H. Cavity plasmonics: large normal mode splitting of electric and magnetic particle plasmons induced by a photonic microcavity. Nano Lett 2010; 10: 4394-4398.

19 Marocico CA, Knoester J. Intermolecular resonance energy transfer in the presence of a dielectric cylinder. Phys Rev A 2009; 79: 053816.

20 Novotny L, Hecht B. Principles of Nano-Optics. Cambridge: Cambridge University Press. 2006.

21 Törmä P, Barnes WL. Strong coupling between surface plasmon polaritons and emitters: a review. Rep Prog Phys 2015; 78: 013901.

22 Tavis $\mathrm{M}$, Cummings FW. Exact solution for an $\mathrm{N}$-molecule radiation-field Hamiltonian. Phys Rev 1968; 170: 379-384.

23 Alù $A$, Engheta N. Input impedance, nanocircuit loading, and radiation tuning of optical nanoantennas. Phys Rev Lett 2008; 101: 043901.

24 Bliokh KY, Bliokh YP, Freilikher V, Savel'ev S, Nori F. Unusual resonators: plasmonics, metamaterials, and random media. Rev Mod Phys 2008; 80: 1201-1213.

25 Huang J-S, Callegari V, Geisler P, Brüning C, Kern J et al. Atomically flat singlecrystalline gold nanostructures for plasmonic nanocircuitry. Nat Commun 2010; 1: 150. 
26 Wu XF, Kullock R, Krauss E, Hecht B. Single-crystalline gold microplates grown on substrates by solution-phase synthesis. Cryst Res Technol 2015; 50: 595-602.

27 Schmidt O, Bauer M, Wiemann C, Porath R, Scharte M et al. Time-resolved two photon photoemission electron microscopy. Appl Phys B 2002; 74: 223-227.

28 Hümmer T, García-Vidal FJ, Martín-Moreno L, Zueco D. Weak and strong coupling regimes in plasmonic QED. Phys Rev B 2013; 87: 115419.

29 Johnson PB, Christy RW. Optical constants of the noble metals. Phys Rev B 1972; 6 : 4370-4379.

30 Jiao LY, Fan B, Xian XJ, Wu ZY, Zhang J et al. Creation of nanostructures with poly (methyl methacrylate)-mediated nanotransfer printing. J Am Chem Soc 2008; 130 $12612-12613$. (c) (i) $\odot$ This work is licensed under a Creative Commons Attributioncc. ${ }_{\mathrm{BY}} \mathrm{NC}$ ND NonCommercial-NoDerivs 4.0 International License. The images or other third party material in this article are included in the article's Creative Commons license, unless indicated otherwise in the credit line; if the material is not included under the Creative Commons license, users will need to obtain permission from the license holder to reproduce the material. To view a copy of this license, visit http:// creativecommons.org/licenses/by-nc-nd/4.0/

(C) The Author(s) 2017

Supplementary Information for this article can be found on the Light: Science \& Applications' website (http://www.nature.com/lsa). 